\title{
Warm-Up Striding Under Load Does Not Improve 5-Km Time Trial Performance in Collegiate Cross-Country Runners
}

\author{
Eric K. O’Neal', Ryan T. Albino', Jonathan C. Swain', Dylan W. Sharp', Tara V. Boy', Lauren \\ G. Killen ${ }^{1}$
}

Affiliations: 'University of North Alabama, Department of Kinesiology, Florence, AL, United States

Correspondence: E. K. O'Neal, University of North Alabama, Faculty for Department of Kinesiology, 1 Harrison Plaza, Florence, AL 35632, United States. Email: eoneal1@una.edu

\begin{abstract}
Post-activation potentiation has proven to be an effective strategy to enhance performance for many tasks, but little research has been conducted specifically concerning endurance sport performance. This study examined whether $5-\mathrm{km}$ run performance could be improved by completing pre-run strides while wearing a $6.8 \mathrm{~kg}$ weighted compression garment (LOAD). A counter-balanced crossover field study design was incorporated with NCAA Division I Cross Country runners $(\mathrm{n}=10)$ during coach-led, official team pre-season "speed day" practices. On Monday of Week 1, testing participants completed a course preview run and strategy session with their coach as they would do in preparation for a meet. The following two Mondays, participants completed the $5-\mathrm{km}$ run as quickly as possible while blinded to pace. The team's habitual warmup routine was used, which included a 3.22-km run followed by a series of dynamic warm-up movements before four, $80-\mathrm{m}$ strides were completed with LOAD or without load (CON). Average wet-bulb globe temperature for both sessions was $22.3^{\circ} \mathrm{C}$. CON did not differ ( $\left.\mathrm{p}>0.05\right)$ from LOAD in split times for kilometres $0.00-1.61$ ( $339 \pm 13$ vs $341 \pm 13 \mathrm{~s}$ ), $1.61-3.22$ ( $312 \pm 15$ vs $312 \pm 16 \mathrm{~s}$ ), $3.22-4.83$ ( $339 \pm 21$ vs $338 \pm 22 \mathrm{~s}$ ), or the 0.17 $\mathrm{km}$ distance kick at the end of the run $(71 \pm 16$ vs $69 \pm 14 \mathrm{~s})$. Overall time was also not improved for LOAD $(1060 \pm 49 \mathrm{~s})$ versus CON $(1062 \pm 55 \mathrm{~s})$. The $\sim 10 \%$ body mass LOAD warm-up strategy failed to improve early, mid-, or finishing kick performance in a 5-km time-trial with well-trained runners.
\end{abstract}

$@$ @JSSMontenegro

POST-ACTIVATION POTENTIATION AND DISTANCE RUNNING

http://mjssm.me/?sekcija=article\&artid=195

KEY WORDS post-activation potentiation, running economy, endurance athletes, leg stiffness

\section{Introduction}

A thorough warm-up (WU) is common, provides a multitude of physiological advantages, and is widely supported for competitive athletes (Bishop, 2003a). However, the facets of optimizing WU for athletic performance are complex, possibly even more so for endurance athletes (Bishop, 2003b). Manipulating non-supramaximal WU activity variables does not appear to improve endurance performance across a variety of exercise modalities. Both the addition of 3 min of higher intensity cycling at the end of a 15-minute WU (Hajoglou et al., 2005) and 15-min steady-state WU running at an intensity of $80 \%$ versus 60 or $70 \%$ of VO2 max (Takizawa, Yamaguchi, \& Shibata, 2018) failed to result in improved cycling or running performance, respectively. Furthermore, a 15 min steady state WU at $75 \%$ versus 55 or $65 \%$ of VO2 max resulted in impaired kayaking performance (Bishop, Bonetti, \& Dawson, 2001). In contrast, shorter supra-maximal intensity WU interventions have produced more promising outcomes, possibly due to post-activation potentiation or improved movement economy. Chronic strength and plyometric style training can enhance distance running performance (Paavolainen, Hakkinen, Hamalainen, Nummela, \& Rusko, 1999; Saunders et al., 2006; Spurrs, Murphy, \& Watsford, 2003), but there are sparse data and a lack of understanding concerning the acute effects of the addition of a supramaximal intensity drill to WU to enhance endurance performance. The substitution of five 10-s sprint intervals during the last $5 \mathrm{~min}$ of WU improved kayak power versus a continuous lower

Received: November 212019 | Accepted after revision: January 172020 | First published online: March 012020

(C) 2020 by the author(s). License MSA, Podgorica, Montenegro. This article is an open access article distributed under the terms and conditions of the Creative Commons Attribution (CC BY).

Conflict of interest: None declared. 
intensity WU-only protocol (Bishop, Bonetti, \& Spencer, 2003), and 4-km cycling performance was improved with the addition of three, 10 -s sprints at $70 \%$ of peak power after 5 min of moderate-intensity cycling versus 6.5 minutes of moderate-intensity pedalling only (Chorley \& Lamb, 2019). Conversely, too high of intensity or duration of priming may also be detrimental to performance. McIntyre and Kilding (2015) found 5 sets of 10-s sprints at maximal effort during a $15 \mathrm{~min}$ warm-up phase produced pre-exercise fatigue levels that impaired 3-km cycling time trial performance compared to lower relative intensity sprint priming bouts.

Two similar supramaximal intensity WU manipulation investigations have also been completed using running modalities. Following a typical WU with jogging and change-of-direction drills, Ingham, Fudge, Pringle, and Jones (2013) had elite runners complete $300 \mathrm{~m}$ of total striding at $800-\mathrm{m}$ race pace. One WU protocol used six, 50-m strides and the other included two, 50-m and one, 200-m strides. In contrast to McIntyre and Kilding (2015), the more demanding protocol with the 200-m run at race pace resulted in improved 800-m run performance. The investigators hypothesized that the enhancement in performance was likely due to increased VO2 capacity during the run related to the doubling in lactate concentration prior to the time trial with the longer duration stride bout versus the shorter distance repeated strides. A limitation of the study of Ingham et al. (2013), was there was no low-intensity-only WU control group for comparison. However, a recent investigation by Gonzalez-Mohino et al. (2018) reported the two different high-intensity WU modalities improved a timed run to exhaustion by approximately 5 and 10\% compared to a low intensity only WU, respectively supporting high intensity striding addition to WU protocols. Interestingly and in opposition to Ingham et al. (2013), the high-intensity WU method with the most improvement were the 6 short duration (6-s) uphill (5\% grade) sprints versus nine, 20 -s strides at the same speed but only a $1 \%$ grade (Gonzalez-Mohino et al., 2018).

Alternatively to altering stride duration or gradient, Barnes, Hopkins, McGuigan, and Kilding (2015) increased WU striding intensity by having runners wear weight vests with a load equivalent to $20 \%$ of body mass during six, 10-s strides. The external loading improved trained runners' time to fatigue on a treadmill protocol of increasing velocity and inclination versus the same WU protocol with unloaded sprints (Barnes et al., 2015). The investigators mechanistically attributed the majority of performance enhancement to increased leg stiffness. These findings suggest a WU alteration to intentionally increase leg stiffness via post-activation potentiation in addition to the traditional thermoregulatory or metabolic alterations typically attributed to WU benefits might be worthy of investigation. The effects of post-activation potentiation on endurance performance are not well documented, but Barnes et al. (2015) have demonstrated a potential benefit for runners by adding loaded strides to their WU regimens. However, we are unaware of any studies that have measured performance under conditions akin to actual racing. Therefore, the purpose of this field-based study was to examine if completing a WU protocol in which striding completed while wearing a weighted compression garment could improve 5-km time trial performance versus unloaded striding in collegiate cross country runners.

\section{Methods}

Participants

Male, NCAA Division I cross-country team runners ( $\mathrm{n}=10 ; 20.2 \pm 2.8$ years) completed all aspects of the current study. In an initial session, after study explanation and confirmed written consent, participants were screened for apparent health risk associated with physical activity (Canadian Society for Exercise Physiology, 1994) and completed a short training history questionnaire. Runners were then assessed for height (177 \pm 6 $\mathrm{cm})$ and body mass $(66.4 \pm 8.5 \mathrm{~kg})$ using a standard stadiometer and digital scale (BWB-800AS, Tanita, Tokyo, Japan). Body fat percentage $(7.7 \pm 2.1 \%)$ was estimated using a three-site skinfold test (Jackson \& Pollock, 1985). Runners then completed a self-selected warm-up followed by a graded exercise treadmill test to determine VO2peak $(61.2 \pm 3.3 \mathrm{ml} / \mathrm{kg} / \mathrm{min})$ in accordance with procedures by Heatherly et al. (2018). In brief, participants began running at a pace $4.02 \mathrm{~km} / \mathrm{h}$ slower than their personal best $5-\mathrm{km}$ pace. Treadmill speed increased by $0.8 \mathrm{~km} / \mathrm{h}$ every 2 minutes until volitional fatigue with treadmill grade maintained at $1 \%$. This study was approved by the University of North Alabama's Institutional Review Board. All participants gave written informed consent prior to data collection.

\section{Experimental Sessions}

The runners reported to the laboratory for three additional sessions during pre-season training approximately one month before the season-opening meet. All sessions coincided with coach-led practices, and multiple facets of the protocol were designed with input from the coaching staff. The experimental performance tests were part of the athletes' "speed day" in the coach-designed, periodized training schedules. All runs took place early in the morning on the same day of the week during the month prior to the start of the collegiate cross-country regular season. Wet bulb globe temperature (Thermalert TH-8, physitemp Inc.) was assessed at the beginning and end of each time trial and, when averaged, was identical between trials at $22.3^{\circ} \mathrm{C}$. Similar "easy" runs were performed the day prior to each time trial. Runners were asked to eat a similar dinner the night before each time trial and were asked to consume a $500 \mathrm{ml}$ bottle of water provided by the investigators before going to bed the night before testing and another $500 \mathrm{ml}$ bottle of water upon waking. Breakfast preference was chosen by each individual runner, and meal choices were replicated if a pre-run meal was consumed. All other beverage intake was ad libitum. No alcohol or substantial caffeine consumption was permitted in 
the $24 \mathrm{~h}$ prior to testing. The first session was simply the teams' traditional WU routine led by coaches and athletes on a rubber turf field and asphalt track followed by a $10 \mathrm{~min}$ break to simulate the "holding" period typically included as part of distance running competitions before a familiarization run session on the 5-km road time trial course. The familiarization trial included the coaching staff running with the team and discussing race strategies for the course as would be completed as part of regular meet preparation. This run was not timed by the investigators. The remaining two trials occurred 7 and 14 days after the first session and were identical with the exception of the type of WU used (described below); the runners completed their second and third runs at maximal effort.

\section{Time trial procedures and course}

The 5-km course selected has been repeatedly used in past investigations from our laboratory and was familiar to the university cross country runners. The first third of the course included two challenging climbs followed by a section of modest rolling hills. The middle third portion of the route was a continual slight descent, and the final third of the course was a continual slight ascent to the finish. The course received minimal vehicular traffic during early morning testing. Members of the investigative team were positioned throughout the course wearing bright clothing and signs to caution drivers that runners were on the road. A traditional cross-country start was used, with runners spaced out along both lanes of the road. After $\sim 200 \mathrm{~m}$, a right turn led to the first hill. Investigators were located at the 1.61-km (1-mile reference for the American runners), $3.22-\mathrm{km}$ (2-mile), 4.83-km (3-mile), and 5-km marks collected splits and overall finishing time on digital stopwatches. All investigators on the course provided consistent verbal support to the runners. To encourage maximal effort and competition, trials were performed with coaches present. Runners were not allowed to wear watches and were not informed of their pace at any point during running. Runners were also not made aware of their finishing times or splits until all trials were completed.

\section{Loading scheme and treatment warm-up protocols}

The experimental WU protocol design matched the habitual WU routine used by the cross-country team members. Runners completed a moderate intensity $3.22 \mathrm{~km}$ run before starting their dynamic movement phase of their WU. This phase included lunge variations, dynamic walking drills, skipping drills, arm swings, carioca, and A, B, and C skips. Immediately after the last skip drills, participants donned the weighted compression garments. Next participants completed four, 80 meter (1 turn) strides with an external load (LOAD) or without an external load (CON) in a counterbalanced crossover design. Loading was accomplished using a unique weighted compression garment (TITIN ForceTM Weighted Shirt System, Titin Tech, USA) (see Figure 1). The garment consisted of an outer short sleeve shirt (87\% Sorbtek, 13\% Lycra) and an inner short sleeve shirt (Pocket Suiri 52\% A.M.Y. 48\% Polyester). Together both shirts weighed $\sim 0.5 \mathrm{~kg}$. The inner shirt had pockets that were loaded with flat, dense gel inserts. The outer compression shirt was used to minimize weight shifting inserts and allow for unrestricted and comfortable movement while under load. There were two chest and upper back pockets, two abdomen and lower back pockets, pockets above each clavicle area, and pockets on the upper arm areas. The pockets held inserts that weighed $\sim 0.2$ or $0.3 \mathrm{~kg}$. Two inserts were inserted into each pocket resulting in a load of approximately $6.8 \mathrm{~kg}(10.4 \pm 1.1 \%$ of body mass).

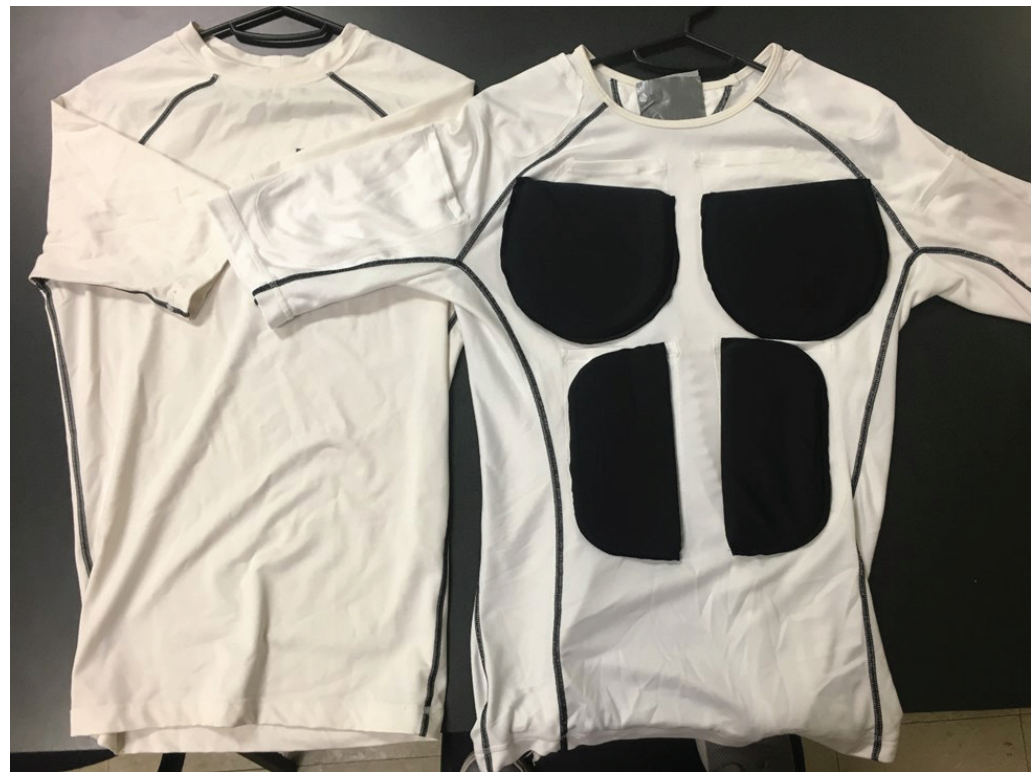

FIGURE 1. Visual representation of the weighted compression garment inner and outer shirts plus inserts. With all pockets loaded with two inserts, the final mass of the weighted compression garment was $6.8 \mathrm{~kg}$.

After the strides, investigative team members helped participants remove the weighted compression garments before runners walked $\sim 200 \mathrm{~m}$ and rested for $10 \mathrm{~min}$ before their time trials, similar to the protocol used 
prior to collegiate cross country competitions. Immediately following the second/final time trial, participants were asked to respond to the following question, "Without knowing your time, how effective do you feel the warm-up with the weight vest was in preparation for running compared to the warm-up without the weight vest?" Runners made a vertical mark on a 100 -mm horizontal line with anchors of "weight vest was much less effective" and "weight vest warm-up was much more effective".

\section{Data analysis}

Paired samples t-tests were used to determine if there were differences between splits and cumulative time at distances of $1.61,3.22,4.83$, and $5.00 \mathrm{~km}$ for CON and LOAD. Pearson's $\mathrm{r}$ was used to analyse the relationship between efficacy belief in the LOAD to improve performance and percentage change in overall 5-km performance. An alpha level of 0.05 was deemed to be significant a priori.

\begin{tabular}{|c|c|c|c|c|}
\hline Cumulative running time (min) & 1.61-km & 3.22-km & 4.83-km & 5.00-km \\
\hline CON & $5.67 \pm 0.24$ & $10.85 \pm 0.50$ & $16.51 \pm 0.89$ & $17.59 \pm 0.92$ \\
\hline$\angle O A D$ & $5.66 \pm 0.18$ & $11.19 \pm 0.75$ & $16.54 \pm 0.69$ & $17.78 \pm 0.81$ \\
\hline Splits (s) & $0-1.61 \mathrm{~km}$ & $1.61-3.22 \mathrm{~km}$ & $3.22-4.83 \mathrm{~km}$ & $4.83-5.00 \mathrm{~km}$ \\
\hline CON & $339 \pm 13$ & $312 \pm 15$ & $339 \pm 21$ & $71 \pm 16$ \\
\hline$\angle O A D$ & $341 \pm 13$ & $312 \pm 16$ & $338 \pm 22$ & $69 \pm 14$ \\
\hline
\end{tabular}

\section{Results}

There were no differences for splits or cumulative time at any marker point (Table 1). Figure 2 displays data for finishing time change percentage and the WU efficacy questionnaire. Under LOAD, five participants improved by $1 \%$ or more; three exhibited less than a $1 \%$ change between treatments, and two runners were slower by more than $1 \%$ (Figure 2). Seven participants reported a modest favouring of LOAD warm-up routine to improve run performance, and the mean WU efficacy scaled displayed a trend of preference for LOAD $(69 \pm 13 \mathrm{~mm})$. However, there was no relationship between change in performance and WU efficacy questionnaire response (Figure 2). Four of the five participants that improved by greater than $1 \%$ under LOAD reported favouring $\mathrm{LOAD}$, but both of the runners that ran greater than $1 \%$ slower under LOAD also reported a favouring of LOAD.

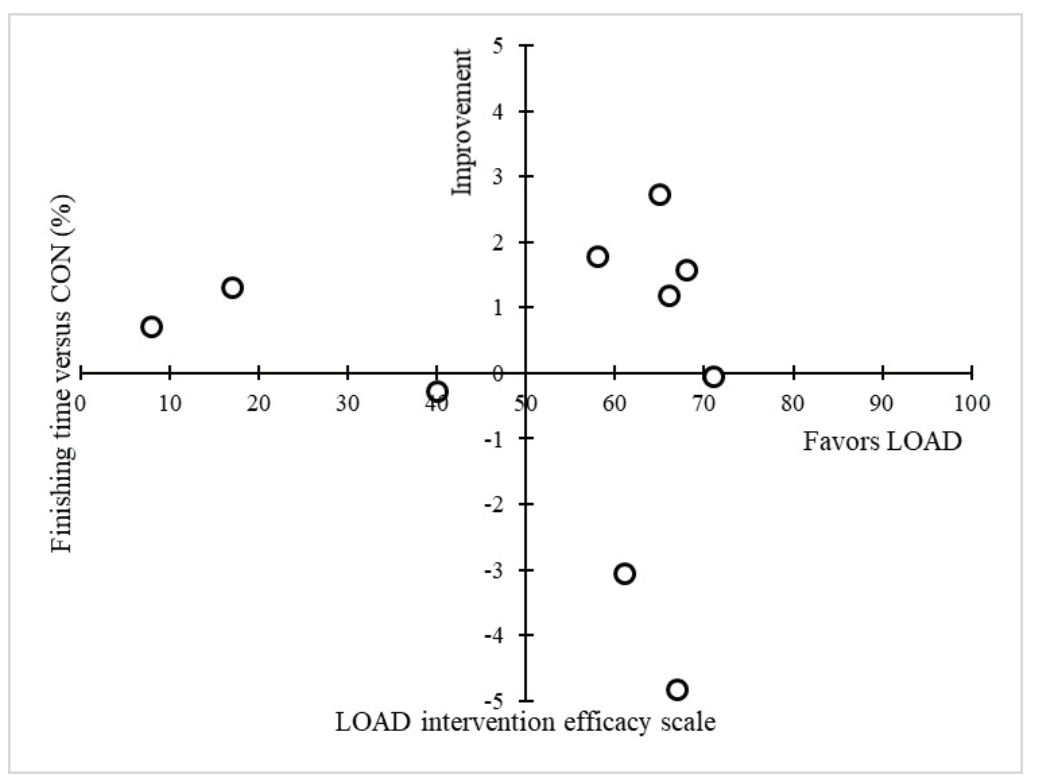

FIGURE 2. Relationship between percentage change in overall finishing time versus CON and response to, "Without knowing your time, how effective do you feel the warm-up with the weight vest was in preparation for running compared to the warm-up without the weight vest?". Runners made a vertical mark on a 100-mm horizontal line with anchors of "weight vest was much less effective" (0) and "weight vest warm-up was much more effective" (100). ( $r=-$ $0.18 ; p=0.61$ ).

\section{Discussion}

Growing evidence supports the fact that short, supramaximal drills during WU routines may improve endurance running performance. A unique approach to manipulate WU supramaximal exercise drills was developed by Barnes et al. (2015). The investigators reported that completing strides while wearing a weighted vest led to longer time-to-fatigue capacity during a graded, incremental intensity treadmill task in trained runners. The inspiration for the current field study was to test this finding in a more ecologically valid per- 
formance task. Time trials to exhaustion lack the between-trial consistency of time trials of a set distance on a treadmill (Laursen, Francis, Abbiss, Newton, \& Nosaka, 2007), and overground running on a familiar 5-km course in a competitive group environment has produced time trial replication coefficient-of-variation levels of $<1 \%$ (Hurst \& Board, 2017). The main finding of the current study was that under ideal conditions and with participants closely matching the anthropometric and running capabilities, the performance advantages reported by Barnes et al. (2015) were not manifested (Table 1) in a competitive, road course 5-km time trial scenario despite mostly positive subjective beliefs (Figure 2) that LOAD intervention was efficacious.

Barnes et al. (2015) used a load equal to $20 \%$ versus $~ 10 \%$ of a participant's body mass. The most probable reason our findings differed was potentially due to the reduced load mass stimulus. The major limitation of this field study was no mechanistic outcomes that might affect performance were evaluated. It is plausible that the lesser load failed to promote increased leg stiffness levels conducive to improved running performance. The 6.8-kg load used reached the weighted compression garment's maximal capacity with all pockets filled with two inserts (Figure 1). After completing numerous investigations with both the weighted compression garment and traditional vests with lead weight loading, our experience has anecdotally supported the claim that the weighted compression garment is more accepted in terms of comfort, particularly during running tasks (Lowe et al., 2016; Scudamore et al., 2016). Supporting this conclusion, the weighted compression garment was generally viewed as favourable (Figure 2) compared to the negative race readiness perceptual effect reported by Barnes et al. (2005). Ergonomic fit and comfort could be critical in terms of promoting runners to adopt loaded striding in real-world practice. An additional practical consideration for the amount of load to be used is the transportation of the garment used to produce the load. Nearly all cross-country meets are held away from runners' home training facilities. With a typical high school or collegiate cross-country team made up of 10-20 runners for both sexes, the lighter and more compact weighted compression garments could be more easily transported and used during meets if the lighter weighted compression garments were used.

Addressing stimulus duration, Barnes et al. (2015) had participants perform six, 10-s strides at $1500 \mathrm{~m}$ pace. The distance and number of strides were designed based on the customary team WU protocol of our collegiate cross-country runner participants. While there was a difference in load mass, we do not feel loading exposure duration likely contributed to differences in performance findings. The stride time duration was not assessed in the current study, but the overall duration of Barnes et al. (2015) was likely similar to the current design using four, $80-\mathrm{m}$ strides. Striding duration was similar or longer than the $300 \mathrm{~m}$ of total striding in (Ingham et al., 2013) or six sets of 6-s strides used by Gonzalez-Mohino et al. (2018).

An important takeaway from Barnes et al. (2015) was that almost all improvement in the run-to-exhaustion time was related to increases in leg stiffness, not running economy. We are unaware of any literature that establishes a minimum threshold for increasing leg stiffness in distance runners. Quantifying this threshold and possibly looking at other methods to increase leg stiffness will be key to further defining optimal supra-maximal WU interventions. Subjective assessments of WU efficacy (Figure 2) were not taken until the completion of the final run. Most runners provided favourable opinions for LOAD in contrast to Barnes et al. (2015) who reported runners felt less ready to race after heavier loaded strides. Runners were only asked to complete the 100-mm efficacy scale, but several runners without prompting from investigators commented anecdotally that they felt more "springy" or made similar comments concerning LOAD. With this consideration, increased leg stiffness plausibly could result in a stronger start, but there were no advantages at the 1.61-km mark during the time trials (Table 1). Due to the nature of the run to exhaustion, no early exercise performance advantages were assessable in Barnes et al. (2015) findings, but improvement in overall durations suggests a potential for late exercise performance enhancement following LOAD. However, this again was not the case in the current study with pace for $3.22-4.83 \mathrm{~km}$ or the final $0.17-\mathrm{km}$ kick failing to exhibit an advantage for LOAD (Table 1).

In conclusion, the findings from the recent study by Barnes et al. (2015) reporting increased leg stiffness and improved running economy following striding under load leading to enhanced time to fatigue during a treadmill performance test was not replicated for collegiate cross-country runners during a road course 5-km time trial. Although mostly well-accepted by the runners, this field-based study found adding external loading to normal pre-race striding routine failed to manifest the performance improvements early, mid-race, or during the final kick phase of a competitive race like scenario. If weighted striding is undertaken during WU, a load greater than $10 \%$ body mass is likely needed to enhance running performance capacity.

\section{Acknowledgements}

The authors thank the collegiate cross-country runners and their coaching staff for allowing investigators to make the alterations in their normal training schedule that made this study possible.

\section{References}

Barnes, K. R., Hopkins, W. G., McGuigan, M. R., \& Kilding, A. E. (2015). Warm-up with a weighted vest improves running performance via leg stiffness and running economy. Journal Science and Medicine in Sport, 18(1), 103-108. doi:10.1016/j.jsams.2013.12.005

Bishop, D. (2003a). Warm up I: potential mechanisms and the effects of passive warm up on exercise performance. Sports Medicine, 33(6), 439-454. doi: 10.2165/00007256-200333060-00005 
Bishop, D. (2003b). Warm up II: performance changes following active warm up and how to structure the warm up. Sports Medicine, 33(7), 483-498. doi:10.2165/00007256-200333070-00002

Bishop, D., Bonetti, D., \& Dawson, B. (2001). The effect of three different warm-up intensities on kayak ergometer performance. Medicine and Science in Sports and Exercise, 33(6), 1026-1032. doi: 10.1097/00005768-200106000-00023

Bishop, D., Bonetti, D., \& Spencer, M. (2003). The effect of an intermittent, high-intensity warmup on supramaximal kayak ergometer performance. Journal of Sports Science, 21(1), 13-20. doi: $10.1080 / 0264041031000070912$

Canadian Society for Exercise Physiology. (1994). PAR-Q and you. Gloucester, Ontario: Canadian Society for Exercise Physiology

Chorley, A., \& Lamb, K. L. (2019). The Effects of a Cycling Warm-up Including High-Intensity HeavyResistance Conditioning Contractions on Subsequent 4-km Time Trial Performance. Journal of Strength Conditioning Research, 33(1), 57-65. doi:10.1519/JSC.0000000000001908

Gonzalez-Mohino, F., Martin, R., Santos-Garcia, D. J., Fidel, P. A., de Asis Fernandez, F., Yustres, I., \& Gonzalez-Rave, J. M. (2018). Effects of High-intensity Warm-ups on Running Performance. International Journal of Sports Medicine, 39(6), 426-432. doi:10.1055/s-0044-102132

Hajoglou, A., Foster, C., De Koning, J. J., Lucia, A., Kernozek, T. W., \& Porcari, J. P. (2005). Effect of warmup on cycle time trial performance. Medicine and Science in Sports and Exercise, 37(9), 1608-1614. doi: 10.1249/01.mss.0000177589.02381.0a

Heatherly, A. J., Killen, L. G., Smith, A. F., Waldman, H. S., Seltmann, C. L., Hollingsworth, A., \& O’Neal, E. K. (2018). Effects of Ad libitum Low-Carbohydrate High-Fat Dieting in Middle-Age Male Runners. Medicine and Science in Sports and Exercise, 50(3), 570-579. doi:10.1249/MSS.0000000000001477

Hurst, P., \& Board, L. (2017). Reliability of 5-km Running Performance in a Competitive Environment. Measurement in PhysicalEducation and Exercise Science, 21(1), 10-14.doi: 10.1080/1091367X.2016.1233421

Ingham, S. A., Fudge, B. W., Pringle, J. S., \& Jones, A. M. (2013). Improvement of 800-m running performance with prior high-intensity exercise. International Journal of Sports Physiology and Performance, 8(1), 77-83.

Jackson, A., \& Pollock, M. L. (1985). Practical assessment of body composition. The Physician and Sports Medicine, 13, 76-90. doi: 10.1080/00913847.1985.11708790.

Laursen, P. B., Francis, G. T., Abbiss, C. R., Newton, M. J., \& Nosaka, K. (2007). Reliability of time-toexhaustion versus time-trial running tests in runners. Medicine and Science in Sports and Exercise, 39(8), 1374-1379. doi:10.1249/mss.0b013e31806010f5

Lowe, J. B., Scudamore, E. M., Johnson, S. L., Pribyslavska, V., Stevenson-Wilcoxson, M. C., Green, J. M., \& O’Neal, E. K. (2016). External loading during daily living improves high intensity tasks under load. International Journal of Industrial Ergonomics, 55, 34-39. doi: 10.1016/j.ergon.2016.07.004

McIntyre, J. P., \& Kilding, A. E. (2015). Effects of high-intensity intermittent priming on physiology and cycling performance. Journal of Sports Science, 33(6), 561-567. doi:10.1080/02640414.2014.960882

Paavolainen, L., Hakkinen, K., Hamalainen, I., Nummela, A., \& Rusko, H. (1999). Explosive-strength training improves $5-\mathrm{km}$ running time by improving running economy and muscle power. Journal of Applied Physiology, 86(5), 1527-1533. doi: 10.1152/jappl.1999.86.5.1527

Saunders, P. U., Telford, R. D., Pyne, D. B., Peltola, E. M., Cunningham, R. B., Gore, C. J., \& Hawley, J. A. (2006). Short-term plyometric training improves running economy in highly trained middle and long distance runners. Journal of Strength and Conditioning Research, 20(4), 947-954. doi: 10.1519/R-18235.1

Scudamore, E. M., Lowe, J. B., Přibyslavská, V., Johnson, S. L., Stevenson, M. C., Langford, T. W., ... O’Neal, E. K. (2016). Three week hypergravity training intervention decreases ground contact time during repeated jumping and improves sprinting and shuttle running performance. International Journal of Exercise Science, 9(2), 149-158.

Spurrs, R. W., Murphy, A. J., \& Watsford, M. L. (2003). The effect of plyometric training on distance running performance. European Journal of Applied Physiology, 89(1), 1-7. doi:10.1007/s00421-002-0741-y

Takizawa, K., Yamaguchi, T., \& Shibata, K. (2018). Warm-Up Exercises May Not Be So Important for Enhancing Submaximal Running Performance. Journal of Strength and Conditioning Research, 32(5), 1383-1390. doi: 10.1519/JSC.0000000000001970 